\title{
On the impact of Masking and Blocking Hypotheses for measuring efficacy of new tuberculosis vaccines
}

Sergio Arregui, Joaquín Sanz, Dessislava Marinova, Carlos Martín, Yamir Moreno

Over the past 60 years, the Mycobacterium bovis bacille Calmette-Guérin (BCG) has been used worldwide to prevent tuberculosis (TB). However, BCG has shown a very variable efficacy in different trials, offering a wide range of protection in adults against pulmonary TB. Previous studies indicate that this failure is related to pre-existing immune response to antigens that are common to environmental sources of mycobacterial antigens and Mycobacterium tuberculosis. Specifically, two different mechanisms have been hypothesized to explain this phenomenon: the masking and the blocking effects. According to masking hypothesis, previous sensitization confers some level of protection against TB that masks vaccine's effects In turn, the blocking hypothesis postulates that previous immune response prevents vaccine taking of a new TB vaccine. In this work we introduce a series of models to discriminate between masking and blocking mechanisms and address their relative likelihood. We apply our methodology to the data reported by BCG-REVAC clinical trials, which were specifically designed for the study of sources of efficacy variability. Our results yield estimates that are consistent with high levels of blocking $41 \%$ in Manaus -95\% C.I. [14\%-68\%]- and 96\% in Salvador -95\% C.I. [52\%-100\%]-). Moreover, we also show that masking does not play any relevant role in modifying vaccine's efficacy either alone or in addition to blocking. The quantification of these effects around a plausible model constitutes a relevant step towards impact evaluation of novel antituberculosis vaccines, which are susceptible of being affected by similar effects if applied on individuals previously exposed to mycobacterial antigens. 
On the impact of Masking and Blocking Hypotheses for measuring efficacy of new tuberculosis vaccines

4 Sergio Arregui ${ }^{1,2}$, Joaquín Sanz ${ }^{1,3,4}$, Dessislava Marinova ${ }^{5,6}$, Carlos Martín ${ }^{5,6,7}$, Yamir Moreno ${ }^{1,2,8}$

5

61 Institute for Biocomputation and Physics of Complex Systems (BIFI), University of Zaragoza, Spain

72 Department of Theoretical Physics, University of Zaragoza, Spain

83 Sainte-Justine Hospital Research Centre, Montreal, Canada.

94 Department of Pediatrics, University of Montreal, Canada.

105 Department of Microbiology, Faculty of Medicine, University of Zaragoza, Spain

116 CIBER Enfermedades Respiratorias, Instituto de Salud Carlos III, Madrid, Spain.

127 Service of Microbiology, Miguel Servet Hospital. IIS Aragon.

138 Complex Networks and Systems Lagrange Lab, Institute for Scientific Interchange, Turin, Italy

14

15

16

17

18

19

20

21

22

23

24

25

26

27

28

29

30

31

32

33

34

35

36

37

38

39

40

41

42

43

44

45

46

47 
48

49

50

51

52

53

54

55

56

57

\section{ABSTRACT}

Over the past 60 years, the Mycobacterium bovis bacille Calmette-Guérin (BCG) has been used worldwide to prevent tuberculosis (TB). However, BCG has shown a very variable efficacy in different trials, offering a wide range of protection in adults against pulmonary TB. One of the most accepted hypotheses to explain these inconsistencies points to the existence of a pre-existing immune response to antigens that are common to environmental sources of mycobacterial antigens and Mycobacterium tuberculosis. Specifically, two different mechanisms have been hypothesized to explain this phenomenon: the masking and the blocking effects. According to masking hypothesis, previous sensitization confers some level of protection against TB that masks vaccine's effects. In turn, the blocking hypothesis postulates that previous immune response prevents vaccine taking of a new TB vaccine. In this work we introduce a series of models to discriminate between masking and blocking mechanisms and address their relative likelihood. We apply our methodology to the data reported by BCG-REVAC clinical trials, which were specifically designed for studying BCG efficacy variability. Our results yield estimates that are consistent with high levels of blocking (41\% in Manaus -95\% C.I. [14\%-68\%]- and 96\% in Salvador $-95 \%$ C.I. $[52 \%-100 \%]-)$. Moreover, we also show that masking does not play any relevant role in modifying vaccine's efficacy either alone or in addition to blocking. The quantification of these effects around a plausible model constitutes a relevant step towards impact evaluation of novel anti-tuberculosis vaccines, which are susceptible of being affected by similar effects, especially if applied on individuals previously exposed to mycobacterial antigens.

\section{INTRODUCTION}

Despite all the efforts in the fight against TB accomplished during the last decades, the disease still remains a major cause of morbidity and mortality worldwide, being responsible for a million and a half deaths per year all around the world (WHO Global Tuberculosis Report, 2014). The increasing emergence of multi drug and extremely drug resistant strains (Dye, Williams \& Williams, 2000) or the association between TB and VIH (Boyle, Lowndes \& Alary, 2002; Korenromp et al., 2003) constitute serious epidemiological threats that evidence the necessity of further public health measures and pharmacological resources against the disease. 
79

89

Among all the possible epidemiological interventions that could contribute to the desired goal of TB eradication, the introduction of a novel preventive vaccine is currently thought to be able to offer the highest and most immediate impact on disease burden reduction. The efficacy of the current TB vaccine BCG is consistent in protecting infants, especially from the most severe forms of meningeal and miliary TB (Mangtani et al) but is limited against pulmonary forms of the disease responsible for transmission fueling the growing epidemic worldwide. Accordingly, nowadays there exist more than fifteen different research teams worldwide developing as many novel experimental vaccine candidates designed as revaccination (boosting) strategies in BCG vaccinated individuals (adolescents or adults) or as a BCG replacement strategies at birth (Marinova et al., 2013).

BCG fails to provide consistent protection to the pulmonary forms of the disease, especially in adults (Rodrigues, Diwan \& Wheeler, 1993), who are the main contributors of overall disease spreading. Consequently, an accurate evaluation of the BCG impact under different conditions - population susceptibility, geography, environmental exposure, etc.- is essential. Such evaluation will allow assessing the efficiency of BCG as a reference vaccine and, at the same time, it will provide new guidelines and methodological tools to better evaluate the potential efficacy of the newly developed TB vaccines. The highly variable and apparently inconsistent results obtained in BCG's efficacy tests and meta-analysis have been subject of intense scientific controversy (Mangtani et al., 2014; Barreto et al., 2014); and the use of BCG during the 20th century has been largely argued (Fine \& Rodrigues, 1990; Bloom \& Fine, 1994).

The hypothesized causes underlying the observed variability of BCG efficacy in different settings include differences between the BCG strains (Oettinger et al., 1999), genetic, epi-genetic or socio-economical differences between populations, study quality, parasitic co-infections, etc (Marinova et al., 2013). In addition, multi-variate meta-analysis of BCG efficacy determination studies consistently determine that latitude is a variable showing a most prominent correlation with BCG performance (Fine \& Rodrigues, 1990; Fine, 1995; Brewer, 2000; Zodpey \& Shrikhande, 2007), pointing to the existence of latitude-driven mechanisms influencing it, rather than other possible explanations related, for example, to the ethnicity of the tested populations (Fine et al., 1999). Among these possible mechanisms, the hypothesis that 
109 agglutinates a greater consensus points to the existence of a complex, latitude-dependent immunological

110 process of environmental sensitization (ES) to mycobacterial antigens which might interfere with the

111 observed action of BCG vaccine in different ways. The hypothesis of ES being the source of BCG efficacy

112 variability has been backed up by different epidemiological observations (Hart \& Sutherland, 1977; Miceli

113 et al., 1988; Al-Kassimi, 1995; Mangtani et al., 2014).

114

115 ES is thought to have its origin in the exposure of individuals either to non tuberculous mycobacteriae

116 (NTM) -whose antigenic similarity to MTB (Chaparas, Maloney \& Hedrick, 1970; Harboe et al., 1979) is

117 able to cause cross reactivity in the human immune system (Black et al., 2001; Weir et al., 2003)-, or to

118 the reservoir of latent infection of MTB itself (and other closely related bacteria within the MTB-complex).

119 Additional sources of sensitization have been postulated, like certain parasitic infections (Ferreira et al.

120 2002). The diversity among the different putative sources of ES is notorious, the relationship between

121 their prevalence and latitude is not homogeneous, and their levels of cross reactivity are variable as well.

122 This situation portraits a complex landscape that makes specially ventured to attribute the geographical

123 patterns of BCG efficacy variation to a single factor, as it could be a global increase in NTM prevalence

124 levels next to the equator (Black et al., 2001; Floyd et al., 2002; Weir et al., 2003), which has been

125 demonstrated to be inaccurate for some species (Hoefsloot et al., 2013). Even though, it seems clear that

126 overall levels of ES increase both with closeness to equator and subjects' age at the time of vaccination.

127

128 Two different mechanisms have been theorized on how this exposition to environmental antigens would

129 affect the response of the host to a vaccine like BCG (Andersen \& Doherty, 2005). The masking

130 hypothesis postulates that ES confers a significant protection against TB in such a way that a vaccine can

131 barely offer an additional level of protection (Palmer \& Long, 1966; Andersen \& Doherty, 2005). As an

132 alternative hypothesis, it has been suggested that ES prior to vaccination may trigger an immune

133 response capable of blocking the assimilation of the vaccine by the host, either if it's a live-attenuated

134 vaccine or if it's a booster. This is known as the blocking hypothesis (Brandt et al., 2002; Andersen \&

135 Doherty, 2005). These two effects have the potential to explain, to a large extent, the variability observed

136 in the trials performed, that is, both the dependence of BCG efficacy on age at the time of vaccination -

137 as an individual gets older its exposition to mycobacteriae increases - and its geographical variations. 
138 Finally, it is worth highlighting that masking and blocking do not exclude each other: in a scenario in which

139 both mechanisms take place, ES would contribute at the same time to reduce disease risk of non

140 vaccinated individuals, and to impair vaccine assimilation of immunized ones.

141

142 Several studies have tackled the problem of evaluating ES impact on BCG vaccine efficacy from different

143 angles. Researches in animal models have shown that environmental mycobacteria strains can interfere

144 with BCG vaccination and with susceptibility to M. tuberculosis infection (Hernandez-Pando et al., 1997;

145 Demangel et al., 2005; Young et al., 2007). The influence of the effects of Masking and Blocking on

146 measurements of efficacy has been also studied from a theoretical point of view (Fine \& Vynnycky, 1998;

147 Mantilla-Beniers \& Gomes, 2009), even though none of these works allows a quantification of masking

148 and blocking effects on vaccine's efficacy levels measured on clinical trials performed on humans.

151 BCG-REVAC trials were designed to discriminate these two effects on BCG performance when applied

152 on individuals of dissimilar ages in the Brazilian cities of Salvador and Manaus (Barreto et al., 2002;

153 Rodrigues et al., 2005; Barreto et al., 2014). In particular, three types of trials were conducted in the

154 study, measuring the efficacy of as many vaccination strategies in each city: newborn vaccination, school-

155 age vaccination and school-age revaccination. The rationale behind the election of such a design is

156 twofold. On the one hand, replicating the experiment design in two cities of the same country located at

157 considerably different latitudes, renders reasonable the assumption that the main source of variability at

158 the efficacies observed is due to different levels of ES, since virtually any other plausible source of

159 variation (i.e. vaccine preparation, strain or application protocol, ethnic diversity etc.) are absent or

160 controlled for across the study. On the other hand, the trials design allows discriminating between

161 blocking and masking effects, since the differences across cities of the efficacies observed for each type

162 of trial are expected to vary depending on what of the two effects is dominant.

163 After the analysis of BCG-REVAC trials, Barreto et al. observed that the efficacy of the vaccine, when

164 applied to newborns and measured later in life did not show a strong geographic variation, which

165 suggests that spontaneous protection related to masking should play a residual role, if any. On the 
166 contrary, when BCG was applied at school age (Barreto et al., 2014), either the first time, or as a second 167 dose, vaccine efficacy observed was in both cases lower in Manaus than in Salvador; which in principle 168 would be compatible with the blocking hypothesis if vaccine assimilation were more efficient in Salvador 169 as a consequence of lower levels of ES bound to its larger distance to the equator However, even if the 170 design of BCG-REVAC trials allowed to qualitatively asses the greater relevance of the blocking 171 mechanism as compared to masking, no actual quantification of these two effects and their relative role

172 has been provided up to now. In this sense, after the work by the BCG-REVAC consortium, several 173 questions remain unanswered, as we do not know 1) what is the relative likelihood of both hypothetical 174 mechanisms when trying to explain the observed results of the trial, 2) how much predictive power would 175 a full model containing both effects gain with respect to single effects scenarios (masking or blocking alone) 3) whether significantly different combinations of masking and blocking strengths could be similarly

177 compatible with the observations derived from the trials, and very relevantly, 4) what are the intensities of 178 blocking and masking effects, and their confidence intervals, yielding a most significant agreement with 179 the data.

181 In this paper, we introduce a family of mathematical models to interpret the results from BCG-REVAC trial 182 under the light of masking and blocking effects, in order to contribute to answering the aforementioned questions within the limitations imposed by the reduced statistical power derived from the reduced

184 number of trials studied. By confronting our model against the results of the BCG-REVAC studies, we are 185 able to measure extent to which these effects are sufficient to explain the efficacies measured (Barreto et 186 al., 2014). Furthermore, we quantify the specific masking, blocking and immunity waning effects yielding 187 best-fitted estimates for the efficacies measured. To this end we compared the likelihoods of three 188 different modeling scenarios: a first model in which both effects concurrently take place, a second model 189 only considering blocking and a third one containing only masking. Translating the trial results into 190 quantitative estimations of blocking and masking strengths constitutes a relevant step towards a deeper 191 knowledge on how BCG efficacy depends on individuals' age and geographical areas. Similarly, it would 192 provide a quantitative reference for the plausible ranges of blocking and masking levels that other TB 193 vaccines might eventually suffer as well; which could be especially relevant in the context of impact 194 evaluation of novel vaccines. Up to our knowledge, the BCG-REVAC studies are the only set of trials 
195 specifically designed to discriminate the effects of Masking and Blocking. Even though our work is

196 restricted to this particular setting, our framework could also be extended to the interpretation of future

197 trials and impact evaluation of future vaccines.

198

199

200

201

202

203

204

205

206

207

208

209

210

211

212

213

214

215

216

217

218

219

220

221

222

223

224

225

226

227

\section{METHODS}

\section{Data analyzed: the Brazilian BCG-REVAC clinical trials}

BCG-REVAC consisted of a set of cluster-randomized trials involving more than 200,000 school-aged children in the Brazilian cities of Manaus and Salvador, whose principal aim was evaluating the effectiveness of BCG under different vaccination protocols.

The enrolled population of the study consisted of non-infected school children between 7 and 14 years old at the moment of randomization. Within this population, individuals presenting a positive BCG scar are separated from the rest, distinguishing, this way, the enrolled individuals who were vaccinated at birth from those who were not. Each group is then split into an intervention and a control group; individuals in the intervention group were vaccinated within the context of the trial. Summing up, there are 4 cohorts in each city: non vaccinated (1), vaccinated after birth (2), firstly vaccinated at school age during the trial (3), and revaccinated, after a first dose applied after birth, in the trial too (4). Upon such classification of enrolled individuals in cohorts, the effectiveness of BCG vaccination strategies was measured by comparing the TB incidence rate within an end-point associated to active disease in the four cohorts, according to three different types of trials: Trial I: BCG at birth vs. no intervention (cohort 2 vs. 1). Trial II: BCG first dose at school age vs. no intervention (cohort 3 vs. 1). Trial III: revaccination at school age vs. first dose at birth only (cohort 4 vs. 2).

\section{A model to describe BCG efficacy variation: masking, blocking and immunity waning.}

The six clinical trials conducted within the framework of BCG-REVAC study -three types of trials per two cities- output efficacies that span from $1 \%$ to $40 \%$ protection (see figure 1 , red continuous lines). In order to explain this variability we propose a model according to which the different protection levels found in each of the four cohorts in the study, schematically shown in figure 2, result from the interplay between the intrinsic vaccine efficacy, its temporal waning patterns, masking and blocking effects. These three 
228 mechanisms of vaccine protection shifts are ultimately responsible for vaccine's performance variation,

229 either in space or in time.

230
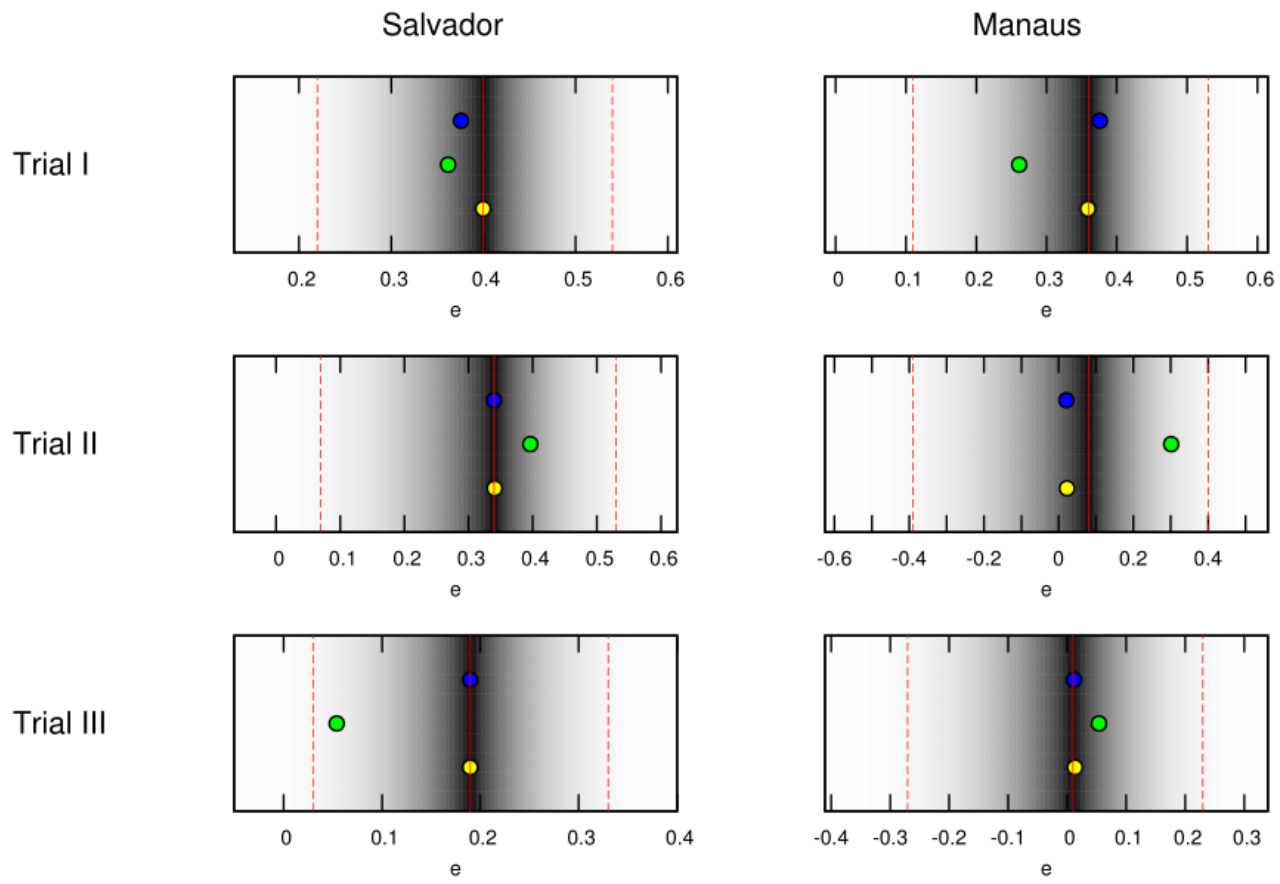

$\begin{array}{r}\text { Model } 1 \\ \text { - Model } 2 \\ \hline\end{array}$

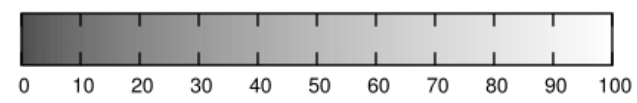

Figure 1: Best fit estimates for each trial by models 1, 2 and 3 (yellow, blue and green dots, respectively) for the trials conducted in the BCG-REVAC study. The colormap represents the probability of obtaining a more extreme value of the efficacy, according to the distributions considered. The probability of zero marks the central estimate (red, continuous line) while the dashed red lines mark the 95\% C.I. reported by Barreto et al. (Barreto et al, 2014). 


\section{NO VACCINE}
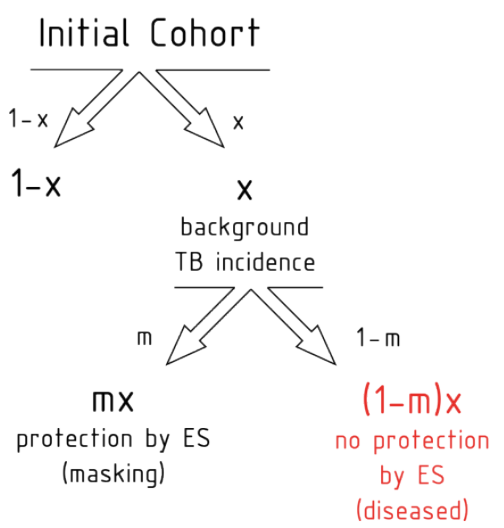

3. SCHOOL AGE VACCINE

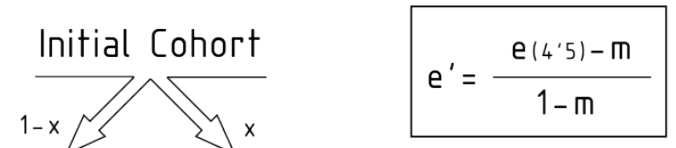

$1-x$

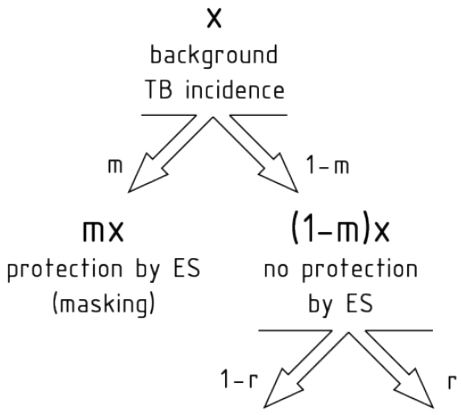

$$
e^{\prime}(1-m) x
$$

tentative protection by vaccine

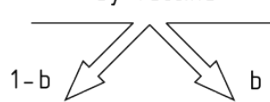

$$
\begin{gathered}
\text { (1-b)e' }(1-m) x \\
\text { protection } \\
\text { by vaccine }
\end{gathered}
$$

243 First of all, in absence of masking or blocking, a naive vaccinated individual will receive a protection level,

244 right after vaccination, that we call $e(0)$. As time after vaccination goes by, this protection level will wane

245 up to $e(t)<e(0)$, generally speaking. This implies that, if we deal with a population in which the

\section{NEWBORN VACCINE}

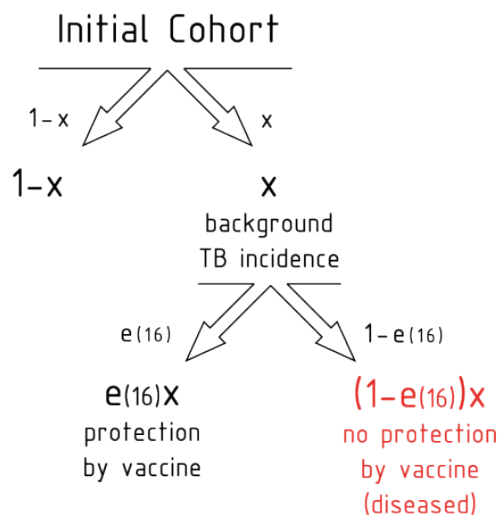

\section{NEWBORN + SCHOOL AGE VACCINES}

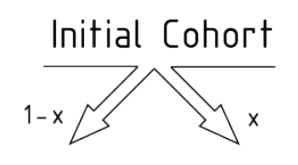

$$
e^{\prime \prime}=\frac{e\left(4^{\prime} 5\right)-e(16)}{1-e(16)}
$$

$1-x$

background TB incidence

$$
\text { e(16) }
$$

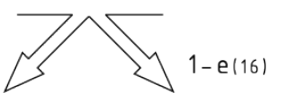

$\mathrm{e}(16) \mathrm{x}$ protection by $(1-e(16)) x$ first vaccine no protection by first vaccine $e^{\prime \prime}$

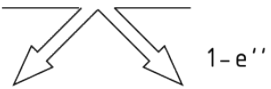

$$
\begin{aligned}
& \text { e"(1-e(16))x } \\
& \text { tentative protection } \\
& \text { by second vaccine }
\end{aligned}
$$
no protection by any vaccine

(1-b)e"(1-e(16))x be" protection by second vaccine protection blocked (diseased)
Figure 2: Scheme of the different contributions to the disease risk for each cohort. 
246 incidence rate of new TB cases per unit time is equal to $x ; t$ years after vaccination this rate is modified

247 to $(1-e(t)) x$, provided that no additional effects take place. Taking that into account, a protective

248 vaccine will have positive efficacy values $e(t) \in(0,1]$, being also possible for a (failed) vaccine to have a

249 negative efficacy if it augments the disease risk among vaccinated individuals instead of reducing it. In

250 our model, the time waning patterns of the intrinsic vaccine efficacy do not depend on the geographical

251 area, but just on time since vaccination, which approximately is, in average, 4.5 years for school age

252 vaccination (cohort 3) and 16 years for newborn vaccination (cohort 2), which implies the consideration of

253 two intrinsic efficacy parameters: $e(4.5)$ and $e(16)$.

254 Besides vaccination, ES can also support protection against disease through the masking mechanism.

255 The masking level, denoted by $m$, is a protection parameter formally equivalent to the intrinsic vaccine

256 efficacy (thus verifying $m \in(0,1]$ for a protective effect, and negative otherwise), whose effects are

257 suffered by initially naive, non-vaccinated individuals subject to ES. Thus, in principle, the longer the time

258 an individual has been exposed to ES -i.e. the older the individual is at the moment of observation-, the

259 higher is the masking-related protection she might show. Masking is also a geography-dependent effect,

260 since it depends on ES, which forces us to consider two masking parameters: $m^{M}$ for Manaus and $m^{S}$

261 for Salvador. The dependence of these parameters on age cannot be resolved, since all the cohorts

262 analyzed in the study have approximately the same age.

263

264 Additionally, if $e(t)$ describes the protection provided by the vaccine to a naive individual in absence of

265 masking or blocking $t$ years after vaccination, we also need to describe how this protection is modified if

266 the vaccine is applied to non-naive subjects. If an individual's immune system has been stimulated prior

267 to vaccination (either by masking like in cohort 3, or by a previous vaccine, like in cohort 4 before the

268 second dose), and consequently she is partially protected against the disease, it is unrealistic to assume

269 that the full effect of the new dose is additive [25]. Instead of that, our model considers that a vaccine

270 dose applied on a previously protected individual will contribute, at most, up to resetting the initial

271 protection levels $e(0)$, provided that no blocking of the vaccine takes place. In cohort 3, this implies that,

272 right after the school age vaccination, if the vaccine is not blocked ( $b=0$, see below), it will have a 
273 protective effect $e^{\prime}$ that will be concurrent with the masking protection $m$ so as to reduce the disease risk

274 from $[1-m] x$ to $\left[1-e^{\prime}\right][1-m] x$. Our estimation of $e^{\prime}$ comes from assuming that such disease risk must

275 equate what we would observe if a vaccine of full efficacy were applied on naive individuals, and

276 observed 4.5 years later:

277

278

$$
\left[1-e^{\prime}\right][1-m] x=[1-e(4.5)] x \rightarrow e^{\prime}=\frac{e(4.5)-m}{1-m}
$$

279

280 Similarly, the school-age dose at cohort 4, will add to the protection provided by the newborn dose $e(16)$, 281 diminishing the disease risk from $[1-e(16)] x$ to $\left[1-e^{\prime \prime}[1-e(16)] x\right.$. To estimate $e^{\prime \prime}$, we assume that, if the

282 second vaccine is not blocked, the disease risk achieved by both vaccines together $[1-e(16)]\left[1-e^{\prime \prime}\right] x$ is 283 equivalent to the disease risk reached by the same vaccine, if applied on unprotected individuals, 4.5 284 years after vaccination:

285

286

$$
[1-e(16)]\left[1-e^{\prime \prime}\right] x=[1-e(4.5)] x \rightarrow e^{\prime \prime}=\frac{e(4.5)-e(16)}{1-e(16)}
$$

287

288

Finally, vaccine intrinsic efficacy can be blocked by prior ES; an effect that we model through the blocking probability $b \in[0,1]$, where $b=0$ means that no blocking appears, while $b=1$ stands for a totally blocked vaccine, meaning that vaccinated individuals would only have the protection level that they already had before vaccination. Blocking is also a geography-dependent factor, since it is considered a consequence of ES as well, which forces us to distinguish $b^{M}$ and $b^{S}$ for Manaus and Salvador, respectively. Unlike masking, blocking does not depend on the age of the individuals at the moment of observation, but on their age at the moment of vaccination. In this case we study cohorts vaccinated at two moments in life -at birth and at the beginning of the trials- being the first of these cases (the newborn vaccination) considered blocking-free, as it is assumed that when the vaccine is applied immediately after birth, there is no place for prior ES. 
299 Taking all these effects into account, we are left with a set of six independent parameters

$300 \stackrel{\sqcup}{P}=\left\{(4.5), e(16), m^{M}, b^{M}, m^{S}, b^{S}\right\}$ to describe the variability observed in the trials, either temporal or

301 geographical, under the light of blocking and masking effects, concurrently. The temporal trends of the

302 level of protection of each cohort are schematically shown in figure 3.

303
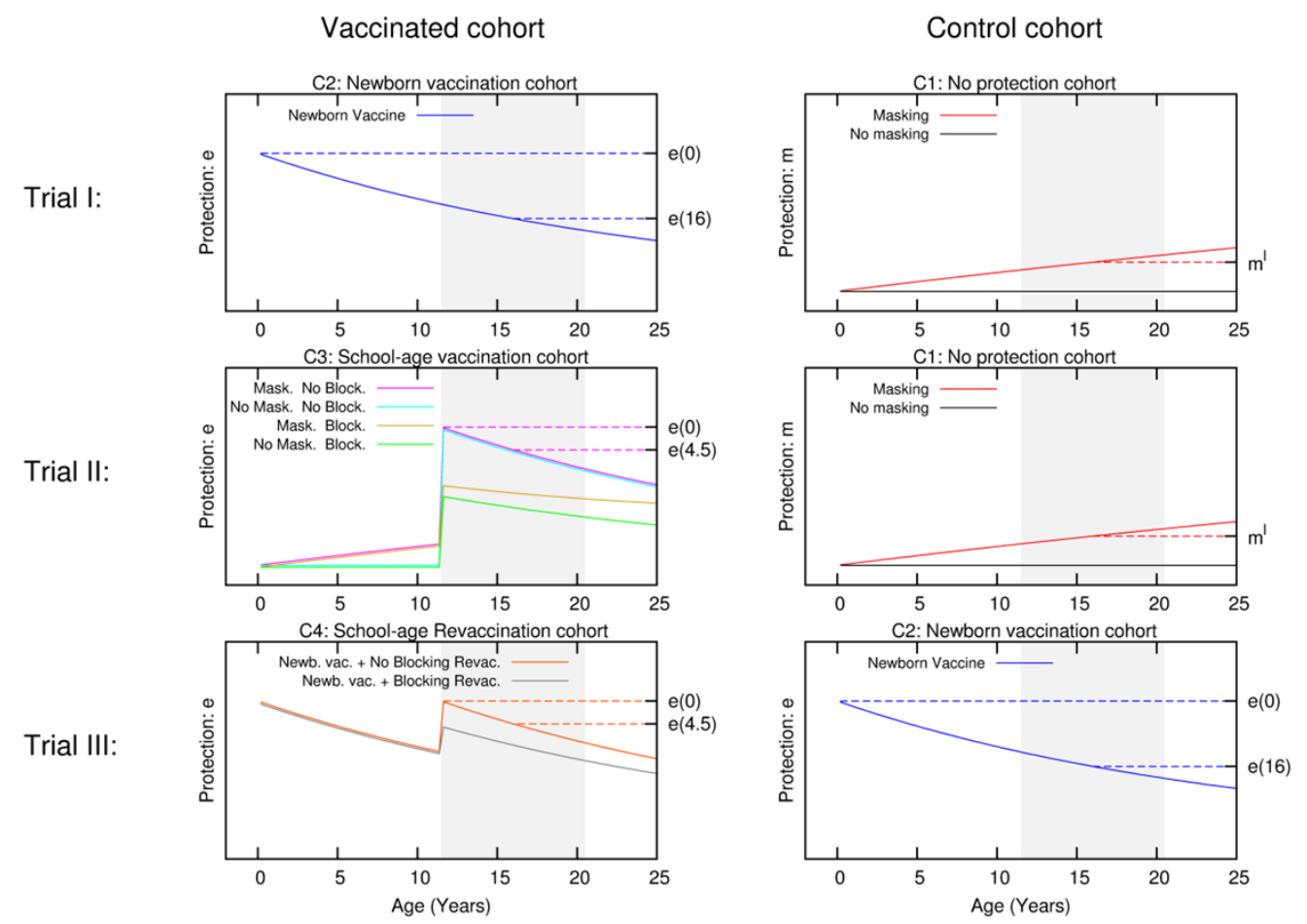

Figure 3: Scheme for the temporal evolution of the level of protection for the cohorts of the three types of trials considered in the work, according to the different vaccination strategies and ES mechanisms. Trial I: The control group is cohort one that corresponds to non-vaccinated individuals. In this cohort, a level of protection above zero can only be due to masking, which is an increasing function with age. In turn, the intervention group corresponds to cohort 2 that is the newborn vaccination group: individuals are vaccinated right after birth, which provides a protection that overcomes any possible masking effect, cannot be blocked by ES and wanes with time. Trial II: The vaccinated cohort is cohort 3, firstly immunized at school age. In this cohort individuals might be protected by masking before the vaccine is applied. Then, at the moment of vaccination, if not blocked, the vaccine will overcome masking protection up to the initial value $e(0)$, which then will wane. Finally, if blocking takes place the protection provided by the vaccine will be reduced. The control cohort in this case is cohort 1 again. Trial III: Intervention group corresponds to cohort 4 , joined by individuals firstly vaccinated at birth, and revaccinated at school age.. At variance to the first dose, which cannot be blocked, the second dose might be blocked by ES or not, in which it will reset the initial protection levels provided by the vaccine. The control group for this trial is cohort 2, that corresponds to individuals only vaccinated at birth. The grey shaded area represents the age window of the individuals enrolled in the study. 
324 disease rates provoked by each effect that takes place in each cohort according to model 1 . Summing all

325 the possible contributions to the development of active disease for each cohort, we derive the general

326 disease rates characterizing each cohort of one city as follows:

327

328

$d_{1}^{l}=\left(1-m^{l}\right) x$

$d_{2}^{l}=[1-e(16)] x$

$d_{3}^{l}=\left(1-b^{l}\right)[1-e(4.5)] x+b^{l}\left(1-m^{l}\right) x$

$d_{4}^{l}=\left(1-b^{l}\right)[1-e(4.5)] x+b^{l}(1-e(16)) x$

329

330

331

where the superscript indicates location, and $x$ the incidence rate observed in the population.

332

333

From (3), it is immediate to derive the expressions for the observed efficacies $\bar{e}$ of each trial according to

334 model 1, which read as:

335

337

338

$M_{1}:\left\{\begin{array}{l}\bar{e}_{I}^{l}=1-d_{2}^{l} / d_{1}^{l}=\frac{e(16)-m^{l}}{1-m^{l}} \\ \bar{e}_{I I}^{l}=1-d_{3}^{l} / d_{1}^{l}=\frac{e(4.5)-m^{l}}{1-m^{l}}\left(1-b^{l}\right) \\ \bar{e}_{I I I}^{l}=1-d_{4}^{l} / d_{2}^{l}=\frac{e(4.5)-e(16)}{1-e(16)}\left(1-b^{l}\right)\end{array}\right.$

339 REVAC trials, which is based on the assumption that the sources of geographical variability for BCG's

340 performance are both masking and blocking effects. From the full model, two reduced versions can be

341 conceived: a masking-free model (model 2 in the following) in which $m^{M}=m^{S}=0$, and a blocking free

342 model in which $b^{M}=b^{S}=0$ (model 3). The efficacies associated to each trial, for models 2 and 3

343 straightforwardly read as follows:

344

$$
M_{2}:\left\{\begin{array}{l}
\bar{e}_{I}^{l}=e(16) \\
\bar{e}_{I I}^{l}=e(4.5)\left(1-b^{l}\right) \\
\bar{e}_{I I I}^{l}=\frac{e(4.5)-e(16)}{1-e(16)}\left(1-b^{l}\right)
\end{array}\right.
$$


345

$$
M_{3}:\left\{\begin{array}{l}
\bar{e}_{I}^{l}=\frac{e(16)-m^{l}}{1-m^{l}} \\
\bar{e}_{I I}^{l}=\frac{e(4.5)-m^{l}}{1-m^{l}} \\
\bar{e}_{I I I}^{l}=\frac{e(4.5)-e(16)}{1-e(16)}
\end{array}\right.
$$

347 By considering these three models, our approach allows quantifying and comparing the plausibility of

348 blocking and masking hypotheses to potentially explain the variation in BCG efficacy trials observed in the controlled setup conceived in the BCG-REVAC trials, taking into account the non-linearities associated to each mechanism, which play a central role in the derivation of Equations (4-6).

351

352

353

354

355

356

357

358

Once the empirical distributions have been defined, for each possible set of parameters and for each of

364 the six trials we define the Z-score associated to the model prediction as:

365

366

$$
Z_{i}^{l}(\stackrel{\square}{P})=\left|\frac{\left[\overline{\boldsymbol{e}}_{i}^{l}(\stackrel{\cup}{P})\right]_{\mathrm{hod}}-\left[\overline{\boldsymbol{e}}_{i}^{l}\right]_{B C G-R E V A C}}{\left[\sigma_{i}^{l}\right]_{B C G-R E V A C}}\right|
$$

367

368 where $\left[\sigma_{i}^{l}\right]_{B C G-R E V A C}^{t}$ will take each of its two possible values depending on the sign of 


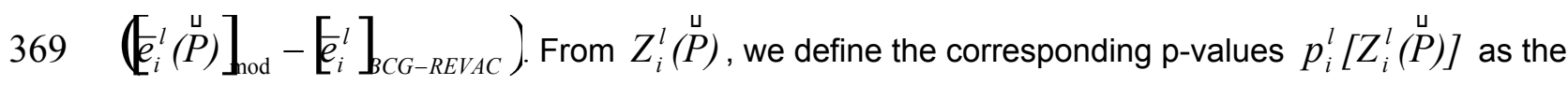

370 probability of the empirical distributions reproducing BCG-REVAC data to have a Z-score $\tilde{Z}$ so that

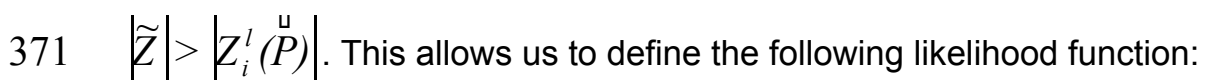

372

373

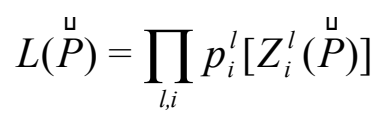

374 to maximize so as to identify the model's parameters $\stackrel{u}{P}^{*}$ more likely to yield the BCG-REVAC results.

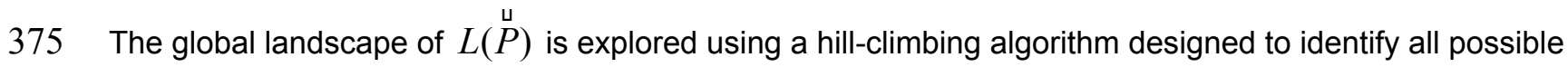

376 local maxima in the space of parameters. Finally, a Levemberg-Marquardt algorithm is used to find a

377 more accurate value of the global maximum, if the latter is unique.

378

379 In order to estimate the confidence interval associated to our model estimation, the following numerical

380 procedure is performed. First, and starting from the maximum likelihood estimate $\stackrel{u}{P}^{*}$, we move on each

381 parameters' axis until a value of $L=0.05$ is reached in each case. We call this increment $A_{j}(j \in[1,6])$

382 (see figure 4). These values are not symmetrical, again, and so we distinguish between $A_{j}^{+}$and $A_{j}^{-}$.

383 Using these asymmetric widths, we construct a two-piece normal distribution for every parameter [30],

384 centered in $\stackrel{u}{P}_{o}$ and having an asymmetric variance given by $\sigma_{j}^{ \pm}=c A_{j}^{ \pm}$, where $c$ is a common

385 modulation coefficient. Besides, the distribution is truncated at 1 . Finally we numerically estimate $c$ by

386 generating sets of points in the parameter space whose coordinates in each axis are obtained from the

387 split normal distributions mentioned for an initial guess of $c$. Through an iterative process we search the

388 value $c=c^{*}$ for which a $95 \%$ of the points generated in the parameters space, yield efficacy estimations

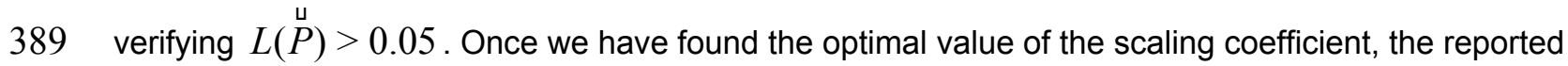

390 uncertainty of the j-th parameter corresponds to 95\% C.I. given the distributions we have used. 
391

392

393

394

395

396

397

398

399

400

401

402

403

404

405

406

407

408

409

410

411

412

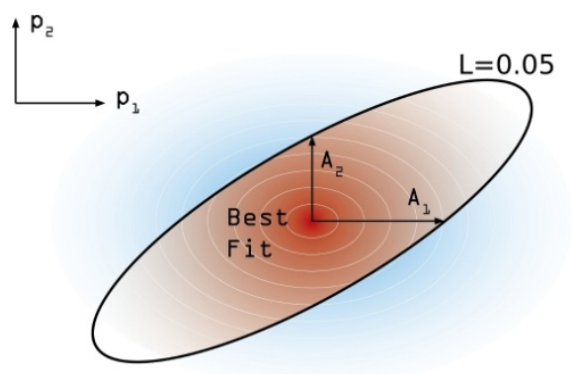

Figure 4: Confidence intervals estimation scheme. Degraded shades represent the joint probability density associated to the estimation of confidence intervals around the model best fit. The modulation coefficient $c$ is determined so as to make the brown area within the black line of $L(\stackrel{\cup}{P})=0.05$ to precisely accumulate the $95 \%$ of the total joint probability distribution.

\section{RESULTS}

In order to find the sets of parameters yielding best estimates of BCG efficacies according to our models we have performed a series of numerical optimization procedures seeking for likelihood maximization. First, we are interested in addressing whether a unique likelihood maximum exists across the parameter space of each model or whether, on the contrary, there exist multiple parameter combinations associated

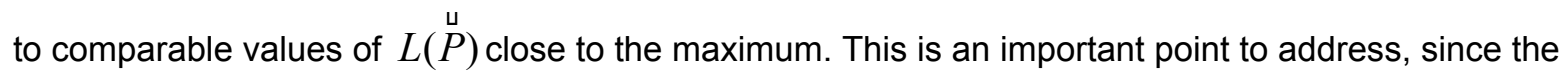
existence of different maxima in a model would indicate the inability of the model to univocally quantify the effects causing the efficacy variations observed. To tackle this question, we performed an iterative hill-climbing algorithm starting from 20,000 random points across the parameter space for each model. As

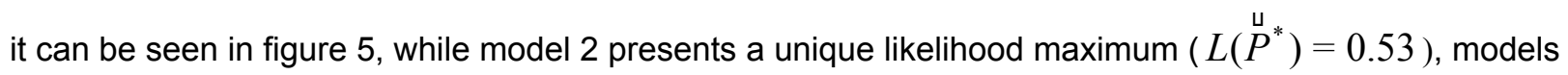
1 and 3 , which contemplates masking, fails at providing a univocal vaccine's description associated to a unique solution from likelihood optimization. 

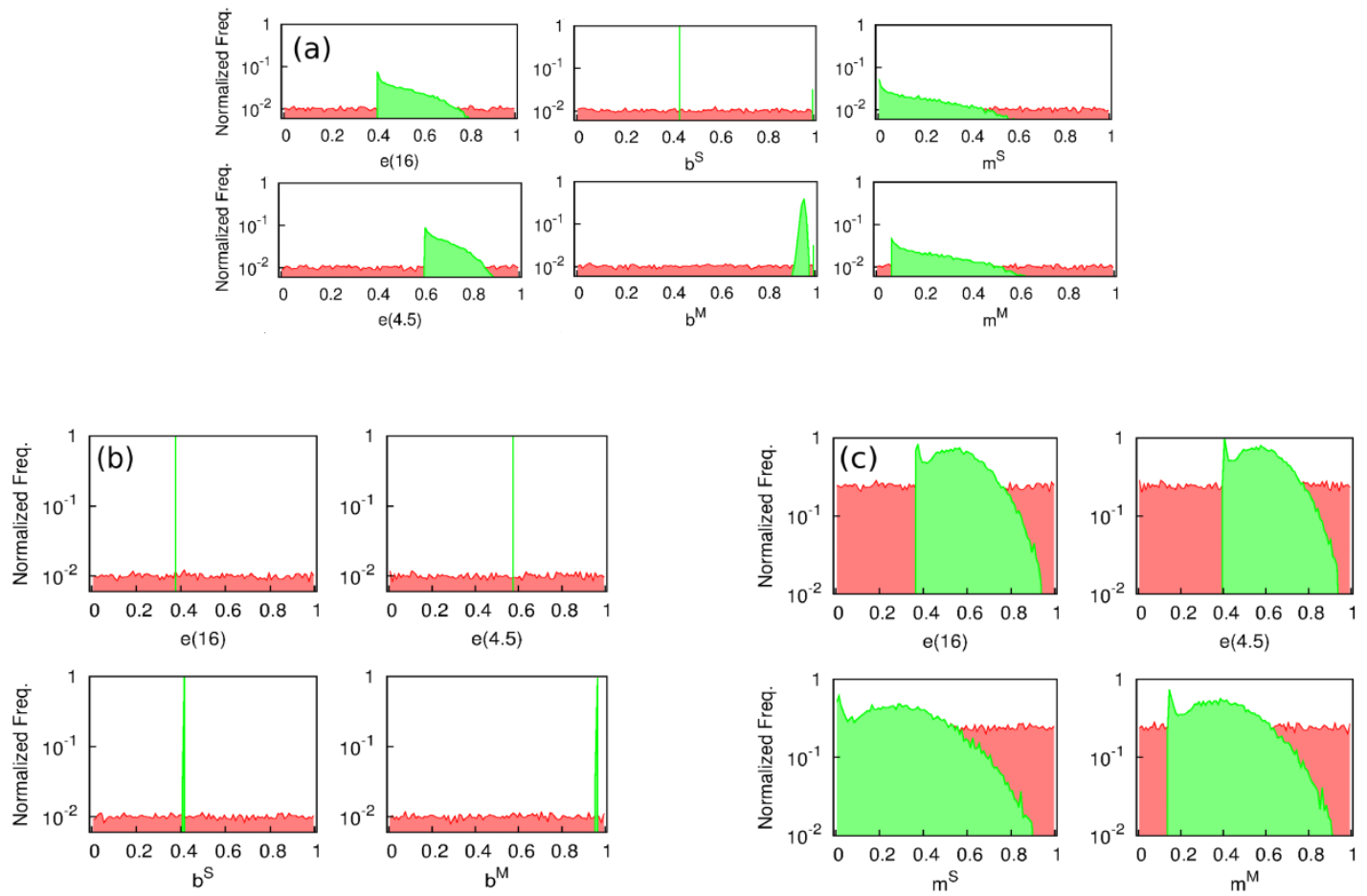

(d)

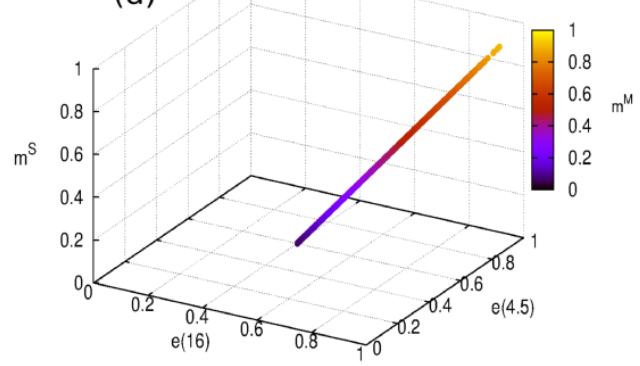

(e)

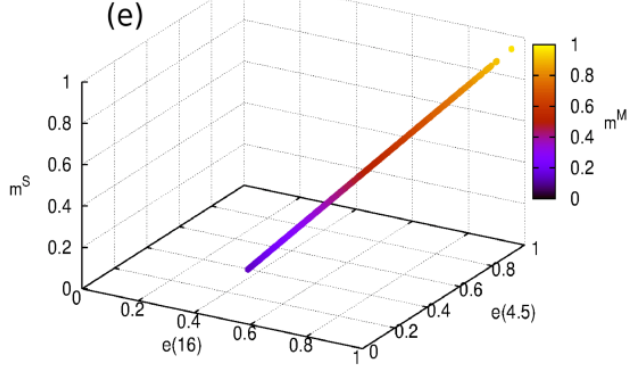

413

414

415

416

417

418

419

420

421

422

423

424

425

426

427

428 Instead of that, as we can see in figure 5, (panels a,c,d and e) models 1 and 3 present a parameters cliff

Figure 5: Panels a,b,c: Hill climbing algorithm distributions for models 1, 2 and 3, respectively. Starting from a series of randomly distributed points in the parameter space (their coordinates distributions are represented in red), a random displacement following a uniform distribution in the parameter space within a hyper-cube of size $d=0.001$ is attempted at each time step, and accepted only if it corresponds to an increasing of the likelihood function $L(\stackrel{\sqcup}{P})$. The algorithm stops when no further move is accepted after $N=10^{7}$ rejected displacements (i.e. the function $L(P)$ reaches a maximum). In green, we see the peaked distribution of the end points of the algorithm around the solution of the models. Panels $d$ and e: parameters cliff yielding quasiconstant values of maximum likelihood $L\left(\stackrel{\cup}{P}^{*}\right)=0.79$ for model 1 and $L\left(\stackrel{\cup}{P}^{*}\right)=0.002$ for model 3. As it can be seen in panel a and $d$, the model versions that contemplate masking are unable to provide a clear univocal vaccine description yielding maximum likelihood. The reason for this behavior is the existence of a region in the parameters space, represented in panel $d$ and $e$, within which, likelihood is almost constant and close to its absolute maximum. 
429 across which, model's likelihood is near to its maximum, and largely comparable $\left(L\left(\stackrel{u}{P}^{*}\right)=0.79\right.$ for

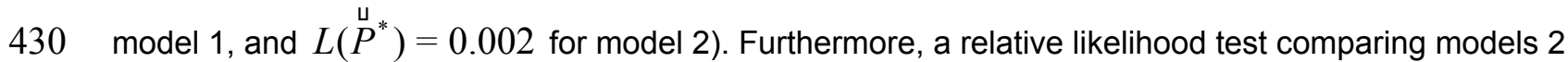

431 and 3 (that is, comparing blocking vs masking as exclusive mechanisms) yields a relative likelihood

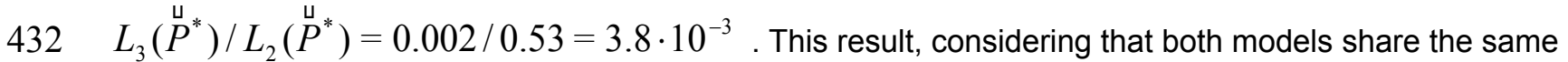

433 amount of parameters, highlights again the inability of masking to provide a picture for vaccine efficacy

434 variation as accurate as blocking does, as we can also see in figure 1, where the best fit provided by each

435 model is presented as well.

436

437 If the analysis of model 3 and its comparison against model 2 allows us to discard masking as an

438 autonomous mechanism able to explain the vaccine efficacy measured in the trials, it remains to be

439 elucidated whether its consideration in addition blocking in model 1 might still be able to significantly

440 improve the fitting of the observed data. To answer this question, we conduct a simple likelihood ratio test

441 in which the null and full models are, respectively models 2 and 1. From such test, we obtain that the

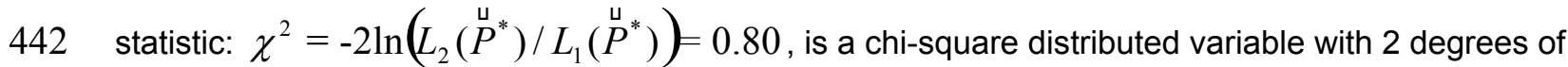

443 freedom (difference between number of parameters of models 1 and 2 ) under the null model. The

444 obtained value does not allow to discard it even with a $50 \%$ confidence $\left(\chi^{2}(p=0.5, d f=2)=1.39\right)$,

445 which indicates that masking is not just unable to provide an acceptable description of the observed data

446 by itself but also makes no significant contribution to explain the variations in vaccine efficacies observed

447 in the trials under study, when considered in addition to blocking. This is also reflected in the close

448 estimates that are found for blocking parameters in models 1 and 2 (see table 1 and figure 5).

449 Besides, if we analyze the combination of parameters that formed the cliff of maximum likelihood in model

4501 , we see that it consists in very similar levels of masking for the two different cities, which enters into

451 conflict with the mentioned correlation between ES effects and closeness to equator.

452

\begin{tabular}{|l|l|}
\hline Parameter & Model 2 (only blocking) \\
\hline$e(4.5)$ & $57.7 \%(46.8 \%$ to $68.6 \%)$ \\
\hline$e(16)$ & $37.6 \%(29.3 \%$ to $45.8 \%)$ \\
\hline$b^{M}$ & $96.4 \%(51.9 \%$ to $99.8 \%)$ \\
\hline$b^{S}$ & $41.1 \%(14.2 \%$ to $68.0 \%)$ \\
\hline
\end{tabular}


454 455 456

459

460

462

463

464

465

466

467

468

469

470

472

473

474

475

476

477

478

479

480

481

Table 1: Optimal parameters of model 2. Models 1 and 3 are unable to provide a unique parameter set yielding maximum likelihood.

In summary, our results point at blocking as the only plausible source of vaccine efficacy variation between the two mechanisms considered, validating the qualitative interpretation of the BCG-REVAC

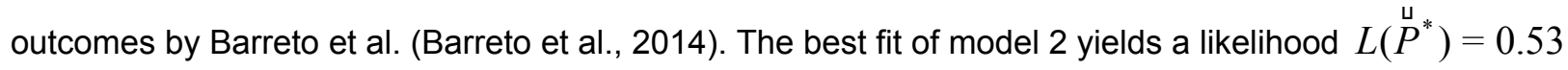
, which corresponds to moderate blocking levels in Salvador $\left(b^{S}=0.41\right.$ c.i. $\left.[0.14,0.68]\right)$ and to almost total blocking in Manaus ( $b^{S}=0.96$ c.i. $\left.[0.52,1.00]\right)$. These results are consistent with the assumed correlation between ES action strength and closeness to equator.

\section{DISCUSSION}

Understanding the mechanisms driving ES effects on BCG performance is a crucial task in the agenda towards the development of new tuberculosis vaccines. In this work, we have proposed a mathematical model that allows the quantitative evaluation of these two effects based on the BCG-REVAC trials performed in Brazil (Barreto et al., 2014). We have seen that the divergence in the measured efficacies of the trials is explained with high values of blocking, which concur with the qualitative discussion made in Barreto, M.L. et al. (Barreto et al., 2014). Furthermore, we have also observed for the first time that no alternative behavior of BCG is compatible with the observed data within the context of a model in which BCG's variability is entirely attributed to ES sensitization.

Admittedly, the range of applications of the results here exposed must be restricted to the provision of a plausible explanation for the efficacy variation patterns observed within a controlled context such as the BCG-REVAC studies, in which the equivalent design of the trials in both cities makes reasonable to assume that ES is the only mechanism responsible for the variations observed. In that sense, the quantitative conclusions reached in this work should be interpreted as a mean to discriminate between the two mechanisms studied when it is reasonable to neglect any source of vaccine variation foreign to ES. Nonetheless, it is worth remarking that our approach cannot provide any insight on the relevance of 
482 ES itself when compared to other plausible sources of variability that could also affect vaccine's efficacy, 483 such as diversity in production, administration, and type of BCG vaccine strain used, as well as the TB

484 strain that circulates in a particular population, among others.

485 In that sense, the analysis of new, hypothetical trials similarly structured, conducted in other geographical 486 areas, could certainly yield different results, If additional sources of variation not considered in this work 487 were playing a relevant role.

488 The model here proposed could however be generalized so as to address some additional questions that go beyond a simple comparison between masking and blocking, which involve a more detailed description of the blocking effect itself.

491

492 On the one hand, it is pertinent to ask whether prior vaccination with BCG might trigger a blocking effect 493 comparable in magnitude to that caused by ES; a question that could be tackled by an extension of the 494 model here proposed in which two blocking parameters -one associated to each source- are considered instead of one. Remarkably enough, distinguishing between BCG vaccine and ES as possibly different causes of blocking might lead to relevant quantitative consequences in what regards impact evaluation of novel vaccines, mostly because the old vaccine is still used in the vast majority of the countries, also in geographical areas in which low levels of ES would be expected.

501 On the other hand, an additional limitation of our study, inherited from the BCG-REVAC studies design, is 502 due to the restriction of trials' endpoints to diseased and not diseased individuals, without measuring 503 infection as a third relevant outcome. This limitation prevents us to address the important question of 504 whether the vaccine is blocked in its protective role against infection, or if, instead, blocking interferes more intensely with the vaccine's performance at reducing the progression rates from latency to active disease (Soysal et al., 2005; Roy et al., 2014).

507 If infection was registered as an additional endpoint in the clinical trials, -something, in general, feasible

508 (Andrews et al., 2015)-, our approach could then be extended so as to estimate two different blocking components associated to the impairment of vaccine's protection against infection and active disease 
510 independently. Once again, such hypothetical study could bring important insights for future vaccine

511 development, and in particular could contribute strongly to the debate of what should be the primary goal

512 of TB vaccines (Hawn et al., 2014). Generally speaking, more studies are needed to evaluate how

513 general are the patterns found by BCG-REVAC trials, with the ultimate goal of assessing a positive

514 explanation to the long lasting problem of BCG efficacy variation patterns.

515

516 CONCLUSIONS

517

518

519

The crucial implications of discriminating and quantifying masking and blocking effects for TB vaccine

520 development are twofold. On one hand, understanding the range, and causes behind variations of BCG

521 efficacy is essential (McShane, 2014), since the efficacy of any novel vaccine will be measured against BCG. On the other hand, depending on where a new vaccine is applied and how old are the target populations, masking or -more likely- blocking effects would affect new vaccines too.

These issues affect different stages of the vaccine development pipeline, as sketched in figure 6 . In the first place, during the process of vaccine evaluation in the context of clinical trials, studies of new tuberculosis vaccines should account for the possibility that prior sensitization may compromise their effects (Mangtani et al., 2014). In this sense, and even if a new vaccine targeting TB in adolescents and adults rather than any other age group is expected to have the quickest impact on disease transmission and control, before we address the question of impact of novel vaccines, it is essential to know if the

531 vaccine is more effective than BCG. The most reliable way of knowing whether a new vaccine works

532 better than BCG is by conducting an efficacy trial in a naive population without previous ES (e.g.,

533 previous BCG vaccination, mycobacterial infection and/or TB contact) in order to avoid possible effects of 534 masking or blocking (Rodrigues, Diwan \& Wheeler, 1993; Andersen \& Doherty, 2005; Barreto et al., 535 2015). 
537

538

539

540

541

542

543

544

545

546

547

548

549

550

551

552

553

554

555

556

557

558

559

560

561

562

563

564

565

566

567

568

569

570

571

\section{TRIAL} DESIGN
VACCINE EFFICACY spreading model
VACCINE

IMPACT

Figure 6: Scheme of the basis for evaluation of anti tuberculosis vaccines in absence of universally reliable protection correlates. First stage: design of vaccine efficacy determination clinical trials: the age of the cohorts must be elected taking into account that prior exposure to mycobacteria -either environmental, M. tuberculosis after exposure or even prior TST or also BCG- may corrupt the observed vaccine efficacies. Second stage: vaccine impact evaluations: bulk, short term and long term impact forecasts should be equally considered, as well as agedistributed impacts in terms of cases, infections and casualties prevented.

Furthermore, and once the efficacy estimation is complete, in order to produce any reliable vaccine impact and cost-effectiveness forecast, modeling scenarios contemplating ES deleterious effects on TB vaccines are mandatory. The fact that, according to our analysis, blocking emerges as the driving effect behind BCG variability poses a potential pitfall to any vaccination strategy focused on individuals older than those analyzed here, including most strategies conceived so far for booster vaccines. This is especially worth noticing because blocking, unlike masking, is not supposed to degrade the vaccineinduced protection obtained further during life by individuals immunized promptly after birth. Again, even if immunizing adolescents is thought to provide better impacts than vaccination strategies focused on younger age-segments, if such a novel vaccine is affected by blocking just as BCG is, then its impact will decrease in a way that, given the high blocking levels here identified, might even revert the comparison. As suggested by Helen McShane "we should optimize deployment of BCG to administration as close to birth as possible" (McShane, 2014). This should be the case for new priming live vaccines candidates based on BCG replacement strategies as well (Marinova et al., 2013).

Taken all together, our results highlight the need for measuring ES effects on novel vaccines performance, as well as of diversifying vaccination strategies.

\section{REFERENCES}

Al-Kassimi FA, Al-Hajjaj MS, Al-Orainey IO, Bamgboye EA. 1995. Does the protective effect of neonatal BCG correlate with vaccine-induced tuberculin reaction? American Journal of Respiratory and Critical 
572

573

574 Andersen P, Doherty TM. 2005. The success and failure of BCG - implications for a novel tuberculosis 575 576

577

578

579

580

581

582

583

584

585

586

587

588

589

590

591

592

593

594

595

596

597

598

599

600

Care Medicine 152, 1575-1578 vaccine. Nature Reviews Microbiology. 3(8), 656-62

Andrews JR, Hatherill M, Mahomed H, Hanekom WA, Campo M, Hawn TR, Wood R, Scriba TJ. The Dynamics of QuantiFERON-TB Gold In-Tube Conversion and Reversion in a Cohort of South African Adolescents. American Journal of Respiratory and Critical Care Medicine. 191, 5 584-591, doi:10.1164/rccm.201409-1704OC

Barreto ML, Rodrigues LC, Cunha SS, Pereira S, Hijjar MA, Ichihara MY, De Brito SC, Dourado I. 2002 Design of the Brazilian BCG-REVAC trial against tuberculosis: a large, simplerandomized community trial to evaluate the impact on tuberculosis of BCG revaccination at school age. Controlled Clinical Trials. 23, $540-553$

Barreto ML, Pilger D, Pereira SM, Genser B, Cruz AA, Cunha SS, Sant`Anna C, Hijjar MA, Ichihara MY, Rodrigues LC. 2014. Causes of variation in BCG vaccine efficacy: Examining evidence from the BCG REVAC cluster randomized trial to explore the masking and the blocking hypotheses. Vaccine 32(30) 3759-3764

Black GF, Dockrell HM, Crampin AC, Floyd S, Weir RE, Bliss L, Sichali L, Mwaungulu L, Kanyongoloka H, Ngwira B, Warndorff DK, Fine PEM. 2001. Patterns and implications of naturally acquired immune responses to environmental and tuberculous mycobacterial antigens in northern Malawi. Journal of Infectious Diseases. 184, 322-329

Bloom BR, Fine PEM. 1994. The BCG experience: implications for future vaccines against tuberculosis. In: Bloom BR, ed. Tuberculosis: pathogenesis, protection and control Washington DC: ASM Press, 531557. 
601 Boily MC, Lowndes C,, Alary M. 2002. The impact of HIV epidemic phases on the effectiveness of core 602 group interventions: insights from mathematical models. Sexually Transmitted Infections 78, i78-i90 603

604 Brandt L, Cunha JF, Olsen AW, Chilima B, Hirsch P, Appelberg R, Andersen P. 2002 Failure of the 605 Mycobacterium bovis BCG vaccine: some species of environmental mycobacteria block multiplication of BCG and induction of protective immunity to tuberculosis. Infection and Immunity. 70, 672-678

607

608

Brewer TF. 2000. Preventing Tuberculosis with Bacillus Calmette-Guérin Vaccine: A Meta-Analysis of the

Literature. Clinical Infectious Diseases. 31, 63-67

610

611 Chaparas SD, Maloney CJ, Hedrick SR. 1970. Specificity of tuberculins and antigens from various

612 species of mycobacteria. American Review of Respiratory Diseases. 101, 74-83

613

614

Demangel C, Garnier T, Rosenkrands I, Cole ST. 2005. Differential Effects of Prior Exposure to

615

Environmental Mycobacteria on Vaccination with Mycobacterium bovis BCG or a Recombinant BCG

616 Strain Expressing RD1 Antigens. Infection and Immunity. April 2005 vol 73 no 4 2190-2196, doi:

617

10.1128/IAI.73.4.2190-2196.2005

618

619

620

Dye C, Williams BG, Williams S. 2000. Criteria for the control of drug-resistant tuberculosis. Proceedings

of the National Academy of Sciences 97, 14, 8180-8185

622

623 Ferreira, AP, Aguiar AS, Fava MWB, Corrêa JOA, Teixeira FM, Teixeira HC. (2002). Can the Efficacy of 624 Bacille Calmette-Guérin Tuberculosis Vaccine Be Affected by Intestinal Parasitic Infections?. Journal of 625 Infectious Diseases, 441-443.

626

627 Fine PEM, Rodrigues LC. 1990. Modern vaccines: mycobacterial diseases. Lancet. 335, 1016-1020

628

Fine PEM. 1995. Variation in protection by BCG: implications of and for heterologous immunity. Lancet.

630

346, $1339-1345$

631 
632 Fine PEM, Vynnycky E. 1998. The effect of heterologous immunity upon the apparent efficacy of (e.g.

633 BCG) vaccines. Vaccine 16, 20, 1923-1928

634

635

636 Fine P, Carneiro A, Milstien J, Clements C. 1999. Issues relating to the use of BCG in immunization

637 programmes. Technical Document from Geneva: WHO

638

639

Floyd S, Pönnighaus JM, Bliss L, Nkhosa P, Sichali L, Msiska G, Fine PEM. 2002 Kinetics of delayed-

640 type hypersensitivity to tuberculin induced by bacille Calmette-Guérin vaccination in northern Malawi.

641 Journal of Infectious Diseases 186, 807-814

642

643

Harboe M, Mshana RN, Closs O, Kronvall G, Axelsen NH. 1979. Cross-reactions between mycobacteria.

644

II. Crossed immunoelectrophoretic analysis of soluble antigens of BCG and comparison with other mycobacteria. Scandinavian Journal of Immunology. 9, 115-124

646

Hart PD, Sutherland I.1977. BCG and vole bacillus vaccines in the prevention of tuberculosis in

648 adolescence and early adult life. Brittish Medical Journal 2, 293-295

649

650

Hawn TR, Day TA, Scriba TJ, Hatherill M, Hanekom WA, Evans TG, Churchyard GJ, Kublin JG, Bekker

651

LG, Self SG. 2014. Tuberculosis Vaccines and Prevention of Infection. Microbiology and Molecular

652

Biology Reviews. 78, 4, 650-671, doi:10.1128/MMBR.00021-14

653

654

Hernandez-Pando R, Pavön L, Arriaga K, Orozco H, Madrid-Marina V, Rook G. 1997. Pathogenesis of

655 tuberculosis in mice exposed to low and high doses of an environmental mycobacterial saprophyte before

656 infection. Infection and immunity. 65, 8, 3317-3327

657

658 Hoefsloot W. et al. 2013. The geographic diversity of nontuberculous mycobacteria isolated from

659 pulmonary samples: a NTM-NET collaborative study. European Respiratory Journal 42(6): 1604-1613.

660 DOI: $10.1183 / 09031936.00149212$

661 
662 Korenromp, E. L., Scano, F., Williams, B. G., Dye, C., Nunn, P. 2003. Effects of Human

663 Immunodeficiency Virus Infection on Recurrence of Tuberculosis after Rifampin-Based Treatment: An

664 Analytical Review. Clinical Infectious Diseases. 37, 1, 101-112

665

666

667 Mangtani P, Abubakar I, Ariti C, Beynon R, Pimpin L, Fine PEM, Rodrigues LC, Smith PG, Lipman M, 668 Whiting PF, Sterne JA. 2014. Protection by BCG Vaccine Against Tuberculosis: A Systematic Review of 669 Randomized Controled Trials Clinical Infectious Diseases. 58(4): 470-80

670

671 Mantilla-Beniers NB, Gomes MGM. 2009. Mycobacterial ecology as a modulator of tuberculosis vaccine 672 succes. Theoretical Population Biology. 75 (2009) 142-152

673

674 Marinova D, Gonzalo-Asensio J, Aguilo N, Martín C. 2013. Recent development in tuberculosis vaccines.

675 Expert Review Vaccines 12(12), 1431-1448

676

677

McShane H. 2014. Understanding BCG is the key to improve it. Clinical Infectious Diseases $\mathbf{5 8}$ Editorial

678 Commentary

679

680

Miceli I, De Kantor IN, Colaiácovo D, Peluffo G, Cutillo I, Gorra R, Botta R, Hom S, Ten Dam HG. 1988.

681 Evaluation of the effectiveness of BCG vaccination using the case control method in Buenos Aires,

682 Argentina. International Journal of Epidemiology 17, 629-634

683

684

Oettinger T, Jorgensen M, Ladefoged A, Haslov K, Andersen P. 1999. Development of the

685

Mycobacterium bovis BCG vaccine: review of the historical and biochemical evidence for a genealogical

686

tree. Tubercle and Lung Disease. 79, 4, 243-250

687

688 Palmer CE, Long MW. 1966. Effects of infection with atypical mycobacteria on BCG vaccination and 689 tuberculosis. American Review of Respiratory Diseases. 94, 553-568.

690

691 Rodrigues LC, Diwan VK, Wheeler JG. 1993. Protective effect of BCG against tuberculous meningitis and 692 miliary tuberculosis: a meta-analysis. Int J Epidemiol. 22, 1154-1158 
693

694 Rodrigues LC, Pereira SM, Cunha SS, Genser B, Ichihara MY, De Brito SC, Hijjar MA, Cruz AA,

695 Sant'Anna C, Bierrenbach AL, Barreto ML, Dourado I. 2005. Effect of BCG revaccination on incidence of 696 tuberculosis in school-aged chil-dren in Brazil: the BCG-REVAC cluster-randomised trial. Lancet. 366, $697 \quad 1290-1295$

698

699 Roy A, Eisenhut M, Harris RJ, Rodrigues LC, Sridhar S, Habermann S, Snell L, Mangtani p, Adetifa I, 700 Lalvani A, Abubakar I. 2014. Effect of BCG vaccination against Mycobacterium tuberculosis infection in

701 children: systematic review and meta-analysis. Brittish Medical Journal. 349, g4643 doi:

$702 \quad 10.1136 /$ bmj.g4643

703

704 Soysal A, Millington KA, Bakir M, Dosanjh D, Aslan Y, Deeks JJ, Efe S, Staveley I, Ewer K, Lalvani A.

705 Effect of BCG vaccination on risk of Mycobacterium tuberculosis infection in children with househld

706 tuberculosis contact: a prospective community-based study. Lancet. 366, 1443-51, doi:10.1016/S0140-

707 6736(05)67534-4

708

709

Wallis KF. 2014. The two-piece normal, binormal, or double Gaussian distribution: its origin and

710 rediscoveries. Statistical Science. 29 (1) 106-112

711

712 Weir RE, Fine PEM, Nazareth B, Floyd S, Black GF, King E, Stanley C, Bliss L, Branson K, Dockrell HM.

713 2003. Interferon-gamma and skin test responses of schoolchildren in southeast England to purified

714 protein derivatives from Mycobacterium tuberculosis and other species of mycobacteria. Clinical \&

715 Experimental Immunology 134, 285-294

716

717 World Health Organization. Global Tuberculosis Report 2014. Available at:

718 http://www.who.int/tb/publications/global_report/en/ (Accesed: February 2015)

719 
720 Young SL, Slobbe L, Wilson R, Buddle BM, De Lisle GW, Buchan GS. 2007. Environmental Strains of

721 Mycobacterium avium Interfere with Immune Responses Associated with Mycobacterium bovis BCG

722 vaccination. Infection and Immunity 75, 6, 2833-2840, doi:10.1128/IAI.01826-06

723

724 Zodpey SP, Shrikhande SN. 2007. The geographic location (latitude of studies evaluating protective

725 effect of BCG vaccine and its efficacy /effectiveness against tuberculosis. Indian Journal of Public health.

$72651,4,205-210$

727

728 